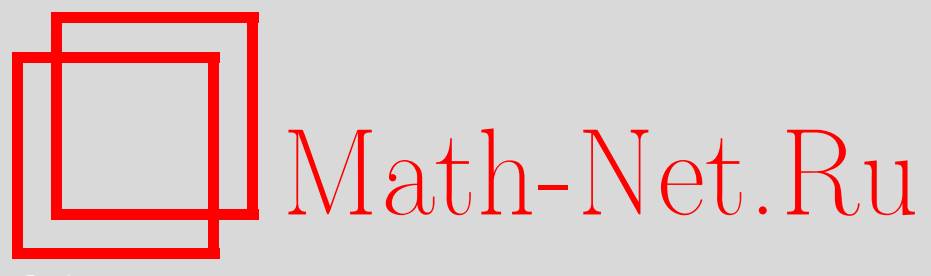

М. А. Горский, Доказательство гипотезы Гала для обобщенных ассоциаэдров серии $D, У M H, 2010$, том 65 , выпуск $6,185-186$

DOI: https://doi.org/10.4213/rm9397

Использование Общероссийского математического портала Math-Net.Ru подразумевает, что вы прочитали и согласны с пользовательским соглашением http://www . mathnet.ru/rus/agreement

Параметры загрузки:

IP: 54.224 .187 .69

26 апреля 2023 г., 15:22:36

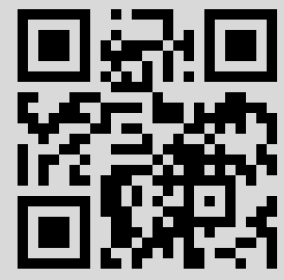




\title{
Доказательство гипотезы Гала для обобщенных ассоциаэдров серии $D$
}

\author{
М. А. Горский
}

Выпуклый многогранник размерности $n$ называется простым, если каждая его вершина принадлежит ровно $n$ гиперграням. Простой многогранник $P^{n}$ называется флаговым, если любое множество его гиперграней $F_{i_{1}}, \ldots, F_{i_{k}}$ имеет непустое пересечение, как только любая пара из этого множества имеет непустое пересечение.

В теории кластерных алгебр определяются (см. [1]) обобщенные ассоциаэдры простые флаговые многогранники, двойственные к кластерным комплексам алгебр конечного типа. Каждый такой многогранник канонически соответствует несвязному объединению диаграмм Дынкина. Диаграммам $A_{n}$ соответствуют обычные ассоциаэдры $A s^{n}$; диаграмма $B_{n}$ и $C_{n}$ - циклоэдры $C y^{n}$. Будем называть серией $D^{n}$ обобщенные ассоциаэдры, отвечающие диаграммам $D_{n}$.

Пусть $f_{i}$ - количество $i$-мерных граней $n$-мерного многогранника $P$. Как обычно, пусть $f(P)(t)=\sum_{i=0}^{n} f_{i} t^{i}$ и $h(P)(t)=f(P)(t-1)-h$-полином. Для простых многогранников выполнены соотношения Дэна-Соммервиля (см. [2]), равносильные возвратности $h$-полинома, т. е. $h(P)(t)=\sum_{i=0}^{[n / 2]} \gamma_{i} t^{i}(1+t)^{n-2 i}$. Полином $\gamma(P)(\tau)=\sum_{i=0}^{[n / 2]} \gamma_{i} \tau^{i}$
называется $\gamma$-полиномом.

ГиПотеза 1 (С.Р. Гал, [3]). Пусть многогранник $P$ флаговый, тогда $\gamma_{i}(P) \geqslant 0$.

Рассмотрим множество $\mathscr{P}^{\text {cube }}$ многогранников, получающихся из куба последовательными срезками граней коразмерности 2. Гипотеза Гала доказана для всех флаговых нестоэдров в [4] с использованием двух фактов: любой флаговый нестоэдр лежит

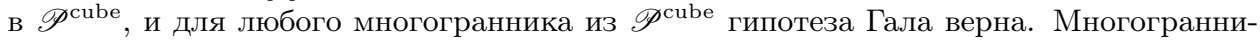
ки $A s^{n}$ и $C y^{n}-$ нестоэдры [5], поэтому для них гипотеза уже доказана. Основной результат нашей работы: многогранники $D^{n}$ не являются нестоэдрами $($ при $n \geqslant 4)$, однако лежат в $\mathscr{P}^{\text {cube }}$, следовательно, гипотеза Гала верна и для них.

Производящим множеством на $[n+1]$ называется совокупность $B$ непустых подмножеств множества $[n+1]$ такая, что: 1$)\{i\} \in B$ для всех $i \in[n+1]$; 2) если $S_{1}, S_{2} \in B$ и $S_{1} \cap S_{2} \neq \varnothing$, то $S_{1} \cup S_{2} \in B$.

Пусть $e_{i}$ - векторы стандартного базиса в $\mathbb{R}^{n+1}$. Для каждого $S \in[n+1]$ определим симплекс $\Delta_{S}=\operatorname{conv}\left\{e_{i}, i \in S\right\}$. Нестоэдром $P_{B}$ называется сумма Минковского $\sum \Delta_{S}$ по $S \in B$. Каждый нестоэдр является простым многогранником (см. [5]).

В [6] введено дифференциальное кольцо многогранников $(\mathscr{P}, d)$, где $d P$ - формальная сумма всех гиперграней многогранника $P$. Для нестоэдров имеет место формула

$$
d P_{B}=\sum_{S \in B / B_{\max }} P_{\left.B\right|_{S}} \times P_{B / S}
$$

Здесь $B_{\max }$ - множество элементов $B$, максимальных по включению, $\left.B\right|_{S}=\left\{S^{\prime} \subset S\right.$, $\left.S^{\prime} \in B\right\}, B / S=\left\{S^{\prime}=S^{\prime \prime} \backslash S, S^{\prime \prime} \in B\right\}$.

Нам понадобятся два следующих известных факта.

(i) Множество гиперграней $A s^{n}$ взаимно однозначно соответствует множеству диагоналей $(n+3)$-угольника, причем две гиперграни пересекаются тогда и только тогда, когда соответствующие им диагонали не имеют общих внутренних точек (см. [7]).

(ii) Множество гиперграней $D^{n}$ взаимно однозначно соответствует множеству Diag $\cup D \cup D^{\prime}$, где множество Diag состоит из пар центрально-симметричных (друг другу) диагоналей $2 n$-угольника, не являющихся диаметрами; каждое из множеств $D$ 
и $D^{\prime}$ состоит из диаметров $2 n$-угольника. Удобно считать, что каждый диаметр из $D$ покрашен в один цвет, из $D^{\prime}-$ в другой. Две гиперграни пересекаются, если соответствующие им диагонали либо не имеют общих внутренних точек, либо являются диаметрами одного цвета, либо являются диаметрами разных цветов, соединяющими одну и ту же пару противоположных точек (см. [1]).

Используя эти факты, получаем следующее предложение.

ПредложЕниЕ 1. $d D^{n}=n\left(\sum_{k=0}^{n-3}\left(A s^{k} \times D^{n-k-1}\right)+2 A s^{n-1}\right)$.

Непосредственно из формулы (1) получаем:

ПредЛожение 2. Пусть $P$-n-мерный многогранник, не раскладывающийся в нетривиальное прямое произведение, и пусть у $P$ есть не менее $2 n+3$ гиперграней, не раскладывающихся в нетривиальное прямое произведение. Тогда $P$ - нестоэдр.

Известно, что многогранники $A s^{n}$ не раскладываются в нетривиальное прямое произведение. Отсюда, используя индукцию, мы получаем ввиду предложений 1 и 2 следующий результат.

Теорема 1. Обобщенные ассоциаэдры типа $D_{n}$ nри $n \geqslant 4$ не являются нестоэдрами.

Теорема 2. Обобщенные ассоциаэдры $D^{n}$ лежат в множестве $\mathscr{P}^{\text {cube }}$.

Доказательство проводится индукцией по $n$ : показывается, что $D^{n}$ можно получить из $D^{n-1} \times I$ последовательной срезкой граней коразмерности 2 . Оказывается, что можно срезать сначала некоторые $n-1$ граней у одного из оснований $D^{n-1} \times 0$, затем грань, являющуюся пересечением двух гиперграней (одной новой, другой старой), соответствующих двум диаметрам разного цвета, а затем еще $n-3$ грани у того же основания. Ввиду флаговости многогранника $D^{n}$, его комбинаторная структура определяется попарными пересечениями гиперграней. На языке диагоналей $2 n$-угольника несложно проверить, что мы получим именно требуемый многогранник.

Следствие 1. Гипотеза Гала 1 верна для обобщенных ассоциаэдров $D^{n}$.

ЗАмЕчАниЕ. Используя конструкцию из [8], можно по последовательности срезок многогранника $D^{n-1} \times I$, приводящих к $D^{n}$, получить явное рекуррентное соотношение, выражающее $\gamma\left(D^{n}\right)$ через $\gamma\left(D^{k}\right)$ и $\gamma\left(A s^{k}\right), k<n$. См. вывод такого соотношения для циклоэдра в [8].

Автор выражает благодарность В. М. Бухштаберу за постановку задачи и внимание к работе и В. Д. Володину за полезные обсуждения.

\section{Список литературы}

[1] S. Fomin, A. Zelevinsky, Ann. of Math. (2), 158:3 (2003), 977-1018; arXiv: hep-th/0111053. [2] В. М. Бухштабер, Т.Е. Панов, Торические действия в топологии и комбинаторике, МЦНмО, М., 2004, 272 c. [3] S. R. Gal, Discrete Comput. Geom., 34:2 (2005), 269-284; arXiv: math/0501046. [4] В. Д. Володин, УМН, 65:1 (2010), 183-184. [5] A. Postnikov, Int. Math. Res. Not. IMRN, 2009, №6, 1026-1106; arXiv: math.CO/0507163. [6] В. М. Бухштабер, Тр. МИАН, 263, Наука, М., 2008, 18-43. [7] C. W. Lee, European J. Combin., 10:6 (1989), 551-560. [8] V. M. Buchstaber, V. Volodin, Upper and lower bound theorems for graph-associahedra, arXiv: abs/1005.1631.

М. А. Горский (М. А. Gorsky)

Московский государственный университет им. М. В. Ломоносова

E-mail: mike.gorsky@gmail.com
Представлено В.М.Бухштабером Принято редколлегией 19.11.2010 